Geograficando, vol. 13, n. ${ }^{\circ}$ 2, e025, diciembre 2017. ISSN 2346-898X

Universidad Nacional de La Plata.

Facultad de Humanidades y Ciencias de la Educación.

Departamento de Geografía

\title{
Tierra vacante y proceso de expansión urbana en las periferias del Partido de La Plata
}

\section{Vacant land and urban expansion process in peripheries of La Plata District}

\section{Daniela Cortizo}

Instituto de Investigaciones y Políticas del Ambiente Construido. Facultad de Arquitectura y Urbanismo. Universidad Nacional de La Plata, Argentina I cortizodaniela@gmail.com

\section{PALABRAS CLAVE}

Tierras Vacantes

Proceso de expansión urbana

Periferias fragmentadas

Partido de La Plata

KEYWORDS

Vacant Land

Urban expansion process

Fragmented Peripheries

La Plata District

\section{RESUMEN}

El objetivo del presente artículo es aportar una visión integral de la problemática de la tierra vacante en el Partido de La Plata, introduciendo variables de análisis que den cuenta de las características del proceso de expansión urbana en las periferias de la ciudad. La metodología adoptada identifica las tierras vacantes del caso de estudio, para analizarlas según dos variables: las intensidades y formas de ocupación del territorio dominantes, y el marco legal normativo que rige el uso potencial futuro de esas tierras. En función de este análisis, los resultados obtenidos sintetizan aspectos relevantes del actual proceso de expansión de las periferias urbanas, identificando las características del eje de crecimiento en donde se encuentran localizadas, en relación a las formas de ocupación del suelo y a las tierras vacantes intersticiales.

\section{ABSTRACT}

The aim of this article is to provide an integral view of the problem of vacant land in La Plata District, introducing analysis variables that account for the characteristics of the urban expansion process in the peripheries of the city. The methodology adopted identifies the vacant lands in case study, to analyze them according to two variables: the intensity and dominant occupation modes of territory, and the normative legal framework that governs the future potential use of these lands. In function of this analysis, the results obtained synthesize relevant aspects of the current process of expansion of the urban peripheries, identifying the characteristics of the growth axis where they are located, in relation to the forms of land occupation and the vacant interstitial lands. 


\section{Introducción}

En el presente trabajo se pretende indagar acerca de cómo contribuyen las tierras vacantes a la generación de periferias fragmentadas, en el marco del proceso de expansión urbana actual en el Partido de La Plata. Se plantean así los siguientes interrogantes: (i) ¿dónde se localizan las tierras vacantes, en relación a las formas e intensidades de ocupación dominantes, y a los usos potenciales permitidos según la normativa vigente? (ii) estas tierras ¿poseen características diferenciales según el eje de crecimiento en donde estén insertas?; y por último, (iii) ¿cómo contribuye esto a la generación de periferias fragmentadas? A su vez, la hipótesis hace referencia a que las características de las tierras vacantes es diferencial según el eje de crecimiento en donde se localice, en función del proceso de expansión urbana actual y la legislación de usos de suelo vigente, contribuyendo así a la generación de periferias territorialmente fragmentadas.

La mencionada expansión urbana del Partido de La Plata se pone de manifiesto al visualizar que, mientras que en un período de 20 años (1991-2010) el crecimiento poblacional fue del 21\% -de 541.905 hab. a 654.324 hab.- (Censo 2010, INDEC), el área urbana construida tuvo un incremento del 127\% -de 4831.6 ha en 1990 a 11,172.8 ha en 2010-(UTDT-CIPUV, 2013), extendiéndose la ciudad con uso predominantemente residencial y dejando terrenos vacantes intersticiales que no fueron ocupados por diversas razones.

El artículo se desarrolla en tres grandes apartados: en primer lugar, se presentan los conceptos de expansión urbana, periferias y tierras vacantes. En segundo lugar se desarrolla la metodología, en donde se caracteriza el caso de estudio, puntualizando en los ejes de crecimiento urbano y el Código de Ordenamiento; para luego focalizar en las variables de análisis, estudiando las intensidades de ocupación del territorio -identificando tierras vacantes-, los tipos de ocupación residencial y los usos permitidos según cada eje de crecimiento. Por último, los resultados y conclusiones, comparando y relacionando los ejes y la contribución de este proceso de expansión a la fragmentación territorial.

\section{Expansión urbana, periferias y tierras vacantes}

En los años '90, las nuevas formas urbanas en Latinoamérica dieron lugar a la denominada "ciudad fragmentada” -de carácter marcadamente insular, segregativa y divisoria-, con lo que se produjo un cambio de referente de la ciudad latinoamericana, desde la ciudad compacta hacia una ciudad dispersa (Frediani, 2009). Estas ciudades se han caracterizado en las últimas décadas por un crecimiento expansivo desordenado, extendiéndose cada vez más en superficie. Pero es importante entender que el crecimiento expansivo no es el problema en sí mismo, sino el modo en que se da ese crecimiento: con escasa regulación por parte del Estado, con centralidades débiles en las periferias o sin centralidades-, con dependencia funcional del centro urbano, bajas densidades, uso predominantemente residencial, escaso espacio público, y con un avance indiscriminado sobre el territorio rural, dándose este crecimiento en forma dispersa, dejando espacios intersticiales sin uso, en el marco de ciudades desiguales y fragmentadas.

En este marco, existen diversas denominaciones para dar cuenta del suelo sin uso -tierra vacante, vacíos urbanos, baldíos, vacantes latentes, terrain vague-. En el presente trabajo, y basándonos en 
definiciones de Clichevsky (2007), Fausto Brito y Rábago (2001) y Fausto Brito (2005), se entiende por tierra vacante a los terrenos remanentes a la dinámica urbana, que permanecen no utilizados y que pueden localizarse dentro del perímetro de la aglomeración, dentro del perímetro denominado "área urbanizable" -área en donde la legislación vigente permite usos urbanos- o en áreas rurales adyacentes a éstas. Estas tierras pueden ser de propiedad privada, o fiscal (sin uso o que ha sido desafectada de sus anteriores usos), y pueden encontrarse de dos formas: (a) subdivididas en parcelas denominadas "urbanas", o (b) en parcelas rurales, que podrían ser usadas para fines residenciales, industriales, comerciales y de servicios por encontrarse dentro de alguna de las áreas antes mencionadas.

Este fenómeno trae aparejado diversos problemas, entre los que se pueden mencionar los altos costos para el Estado, para la provisión de infraestructura y servicios básicos para nuevas urbanizaciones, el uso masivo del automóvil particular y el consumo excesivo del suelo y de recursos energéticos, entre otros, que aportan a una ciudad cada vez más insostenible.

Estas tierras se encuentran localizadas en gran medida en las periferias urbanas. Para definirlas, es necesario mencionar que existe una nueva concepción de estos espacios: ya no son solo la parte “fea” de las ciudades: a pesar de que posean gran cantidad de características negativas -como el escaso valor de centralidad, la poca integración, la dependencia de otras zonas dominantes dentro del sistema urbano, el bajo nivel de accesibilidad urbana, el déficit infraestructural, la carencia de servicios, equipamientos y centralidad, la especulación abusiva del suelo y la segregación socialque conducen a la calificación de lugares urbanos incompletos (Arteaga Arredondo, 2005), poseen a su vez características positivas, que hacen de las periferias lugares buscados para habitar, por las cualidades medioambientales que el centro ya no posee. Estos espacios atraen por la condiciones de vida que ofrecen, "formando parte de una realidad consolidada que revela un profundo cambio en los hábitos de los asentamientos que caracterizan el panorama urbano y territorial” (González Plazas, 2006).

Las grandes tierras vacantes, los intersticios entre tejidos ya consolidados, la transformación del espacio construido, los usos obsoletos o desplazados hacia la periferia, la identificación de una lógica propia y de una diversidad espacial, se han convertido en las oportunidades de la periferia para repensarla y transformarla (Arteaga Arredondo, 2005). Es por esto que es necesario definirlas como una variedad de lugares que reclaman un conocimiento y una intervención adecuados a sus especificidades.

Asimismo, resulta importante entender que la existencia de las tierras vacantes al interior de estas periferias urbanas no tiene su origen en una sola causa; por el contrario, existen múltiples causas que explican su generación y permanencia, como producto de mercados de suelo no regulados, que están en relación a las características propias de las tierras, vinculadas con las necesidades de la demanda; los ciclos económicos y la dinámica del mercado inmobiliario (en relación a comportamientos especulativos de los agentes productores de suelo); las políticas que impactan en la valorización de espacios urbanos; los factores socioculturales, políticos y legales relacionados con la posesión de inmuebles; como así también problemáticas vinculadas al riesgo ambiental (Fausto Brito, 2005), entre otras. Es por esto que estas tierras no deben entenderse como fragmentos aislados, sino como parte de un sistema, que es la ciudad, y las dinámicas de crecimiento que en ella 
operan.

La existencia de los espacios vacantes al interior de las ciudades presupone la posibilidad de introducir nuevos usos -no sólo viviendas, sino también equipamientos urbanos, reservorios, parques-, implementar programas de desarrollo, planes de rehabilitación (Larangeira, 2004). Si el Estado los regula, pueden convertirse en un recurso para contribuir en la mejora de la calidad de vida y en la integración social, siendo las políticas urbanas la herramienta fundamental que posee, a partir de la cual se diseñan y se aplican instrumentos de regulación púbica de la ciudad, para que ésta no sea un resultado estricto de las lógicas del mercado (Barenboim, 2013). Es por ello que es deber del Estado decidir hacia dónde crece la ciudad, cómo se desarrolla, y de qué forma lo hace, y la normativa de usos de suelo es una herramienta fundamental que tienen los municipios para esto. En este sentido, la cuestión de las tierras vacantes cobra importancia, ya que son los espacios en donde la ciudad va a crecer: de la forma en que se intervenga sobre estas tierras se podrían atenuar algunos de los problemas generados por la ciudad dispersa.

\section{Metodología}

La metodología adoptada identifica las tierras vacantes del caso de estudio -el Partido de La Plata-, para luego analizarlas según dos variables: las formas/intensidades de ocupación dominantes, y el marco legal normativo que rige el uso potencial futuro de esas tierras. Se utiliza un Sistema de Información Geográfica (SIG) para la sistematización y análisis de la información.

El Partido de La Plata, ubicado a $56 \mathrm{~km}$ al sudeste de la Ciudad de Buenos Aires, contiene la ciudad homónima, capital de la Provincia de Buenos Aires. Es la cuarta ciudad más poblada del país y el quinto aglomerado urbano con más habitantes después de Buenos Aires, Córdoba, Rosario y Mendoza. Tiene una población total de 654.324 hab. y una superficie de 88.887 ha (Censo 2010, INDEC). Se distribuyen en 195.443 habitantes que residen en el casco fundacional (2807 ha), es decir el 37,7\% de la población total del partido se localiza en el 3,25\% de la superficie total, que como centro urbano cuenta con todos los servicios e infraestructura, transporte, espacios públicos, equipamientos, etc. Pero la ciudad creció más allá de los límites de la circunvalación, extendiéndose en superficie, a partir de vías estructurales de circulación, pero sin seguir muchos de los parámetros que habían sido establecidos en la planificación de la ciudad. Así, el crecimiento en la periferia se dio de forma desordenada, con bajas densidades, con usos predominantemente residenciales y centralidades débiles, dependiendo del centro urbano y con uso predominante del automóvil. En estas periferias es donde residen los 458.881 habitantes restantes (62,3\% de la población total) en 86.080 ha $(96,7 \%)$. 
Figura 1. Ejes de crecimiento en el Partido de La Plata.

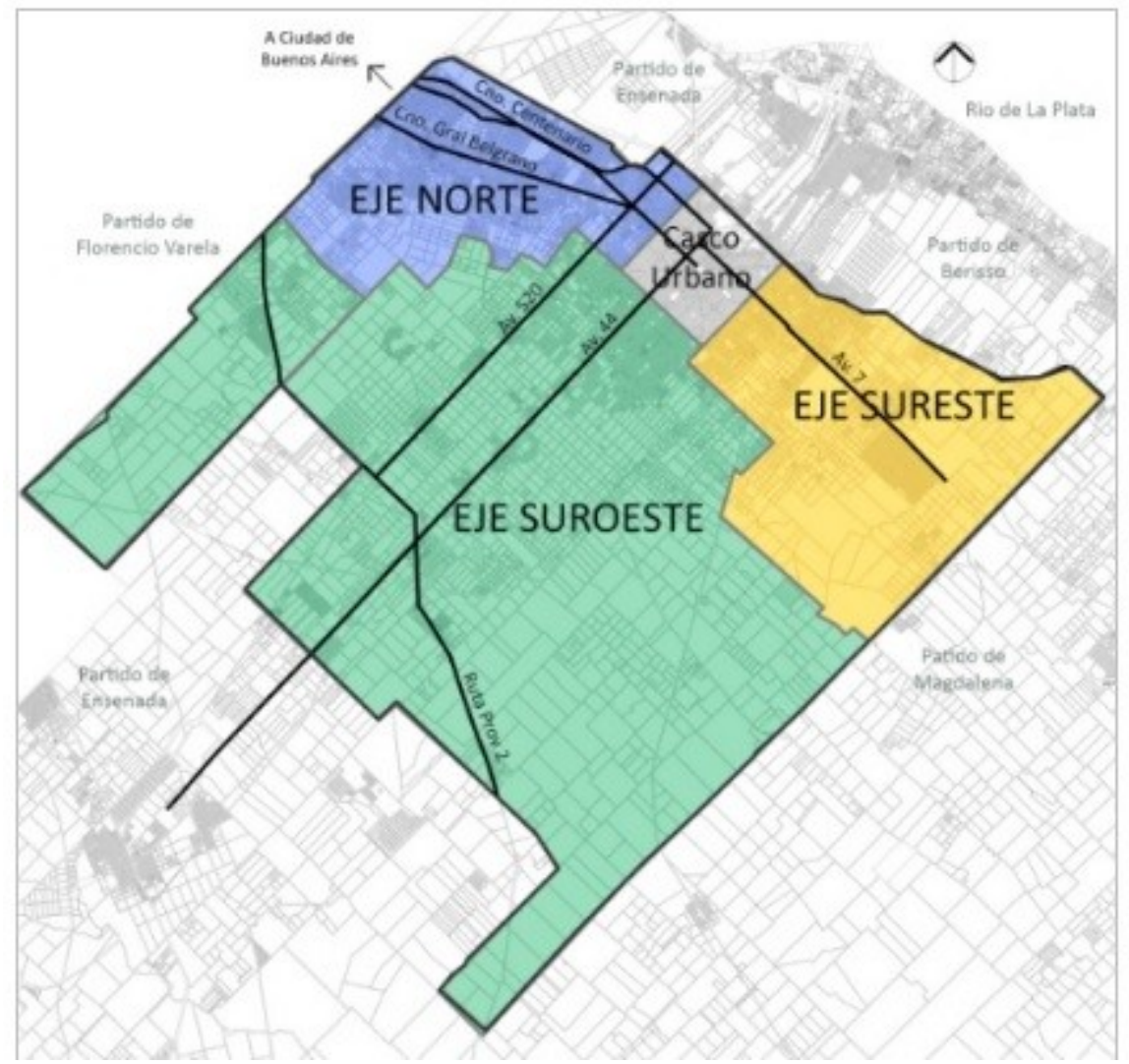

Fuente: elaboración propia

De la configuración urbana del partido se identifican tres ejes de crecimiento (Figura 1). Cada eje ha recepcionado tendencias que definieron los rasgos distintivos desde el punto de vista social y urbano ambiental para cada una de las tres periferias del partido (Rocca y Rios, 2012) (Tabla 1). (i) El Eje Norte, que se encuentra ubicado en relación al Área Metropolitana de Buenos Aires (AMBA), posee tres vías principales que lo estructuran -el Camino Gral. Belgrano y el Camino Centenario, y las vías del Ferrocarril Roca-, formado por las delegaciones de Tolosa, Ringuelet, Gonnet, City Bell, Villa Elisa, Hernández, Gorina y Arturo Seguí; (ii) el Eje Sudeste, cuya vía estructurante es la Avenida 7, y formado por las delegaciones de Villa Elvira, Altos de San Lorenzo y Arana; y, por último, (iii) el Eje suroeste, estructurado por las avenidas 44 y 520, formado por las delegaciones San Carlos, Romero, Abasto, Los Hornos, Olmos, El Peligro y Etcheverry.

\section{Tabla 1. Datos generales por eje de crecimiento}

\begin{tabular}{|c|c|c|c|c|}
\hline & $\begin{array}{c}\text { Superficie } \\
\text { (ha) }\end{array}$ & $\begin{array}{c}\text { Población } \\
\mathbf{2 0 1 0} \text { (hab.) }\end{array}$ & $\begin{array}{c}\text { Necesidades básicas } \\
\text { insatisfechas (NBI) }\end{array}$ & $\begin{array}{c}\text { Crecimiento poblacional } \\
\text { (variación inter censal } \\
\text { 2001-2010) }\end{array}$ \\
\hline Eje norte & 11.219 ha & 170.297 hab. & $7.1 \%$ & $13 \%$ \\
\hline Eje sureste & 16.261 ha & 98.888 hab. & $13.1 \%$ & $12 \%$ \\
\hline Eje suroeste & 58.600 ha & 189.696 hab. & $17 \%$ & $27 \%$ \\
\hline
\end{tabular}

Fuente: Elaboración propia en base a Censos INDEC años 2001 y 2010. 
En relación a los condicionantes para reconocer los espacios vacantes, se debe decir que se trata de tierras difíciles de identificar y cuantificar, ya que muchas de ellas son parcelas dispersas de pequeños tamaños. A su vez, cabe destacar el dinamismo con el que esto se modifica, con el que esta ciudad se expande y estas tierras vacantes se ocupan. Es por ello que para la identificación de tierras vacantes se ha elaborado una metodología basada en la fotointerpretación de imágenes satelitales de Google Earth (año 2016), utilizando la manzana $\left(10.000 \mathrm{~m}^{2}\right)$ como unidad de análisis, clasificándolas según dos parámetros: (i) Intensidad de Ocupación, y (ii) Uso Dominante.

(i) Las intensidades de ocupación están en función de la vacancia de la tierra. Se dividen en 4 tipos, que están en relación a la subdivisión o no de la manzana en parcelas, y al porcentaje de espacio vacante y ocupado (Tabla 2):

- Manzana vacante: terrenos que nunca se usaron ni ocuparon por diversos motivos, tierras productivas que ya no se utilizan, tierras fiscales sin uso actual, cavas sin utilizar, entre otras.

- Manzana ocupada sin subdivisión en parcelas urbanas: predominancia de espacio libre por sobre espacio construido, grandes parcelas con baja ocupación del suelo.

- Manzana subdividida con predominancia de parcelas vacantes: son espacios en consolidación, con un alto porcentaje de lotes vacantes en relación a los ocupados.

- Manzanas subdivididas con predominancia de parcelas ocupadas: son manzanas consolidadas, con poca presencia de tierras vacantes (solo se encuentra alguna parcela aislada, pero predomina la ocupación).

Tabla 2. Manzanas con distintos tipos de intensidades de ocupación

\begin{tabular}{|c|c|}
\hline Características & \\
\hline Manzana vacante & Foto ejemplo \\
\hline $\begin{array}{c}\text { Manzana ocupada sin } \\
\text { subdivisión en parcelas } \\
\text { urbanas }\end{array}$ & \\
\hline $\begin{array}{c}\text { Manzana subdividida con } \\
\text { predominancia de parcelas } \\
\text { vacantes }\end{array}$
\end{tabular}


Fuente: Elaboración propia en base a foto interpretación

(ii) A su vez, se pueden identificar en estas manzanas (y el agrupamiento de ellas) zonas según el uso dominante(Tabla 3): Residencial formal abierto; Residencial formal cerrado; Residencial Informal; Vacante; Productivo; Espacios verdes;Usos específicos.

\section{Tabla 3. Manzanas con distintos tipos de usos dominantes}

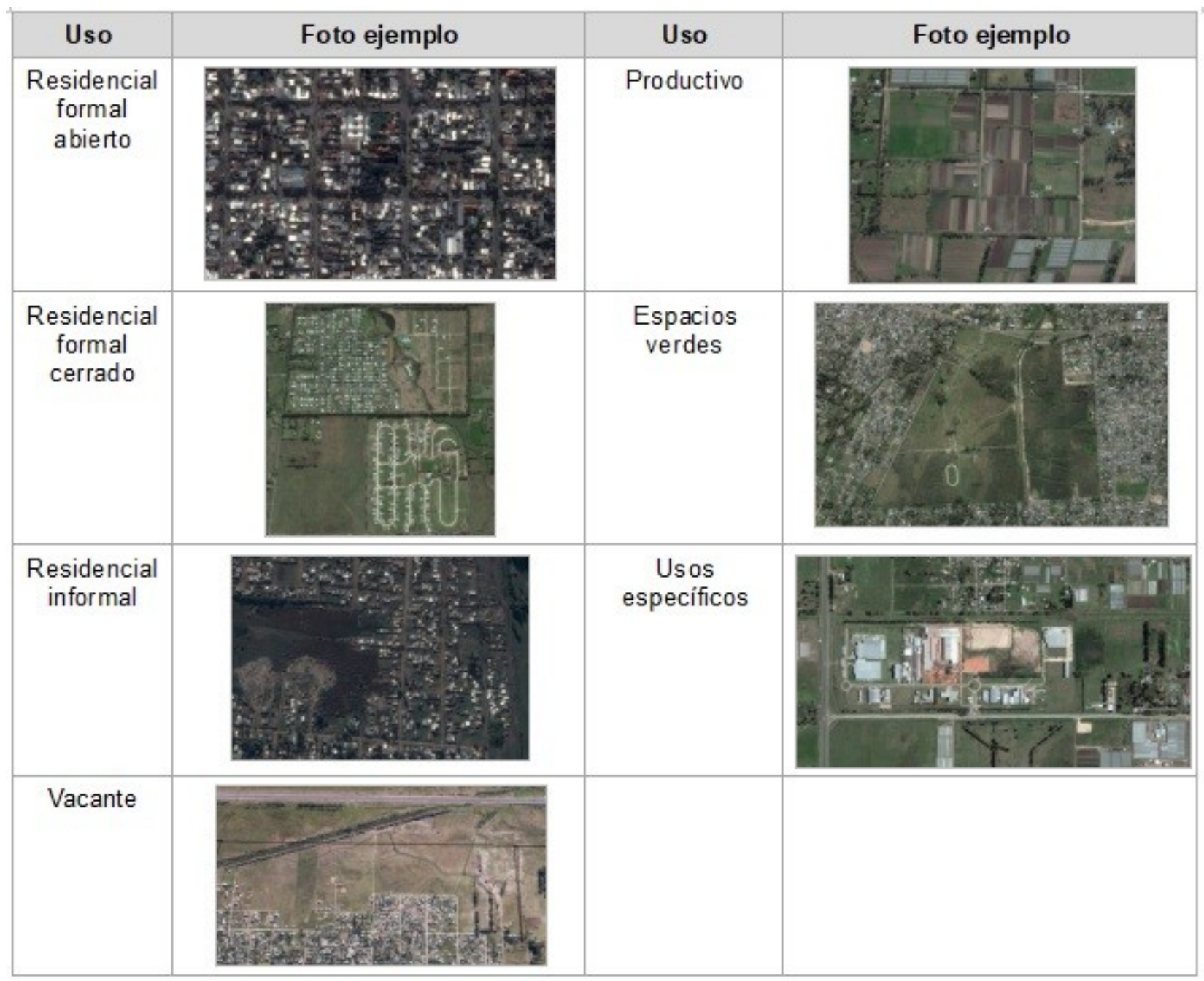

Fuente: Elaboración propia en base a Registro de Villas y Asentamientos del Ministerio de Infraestructura y Servicios Público de la Provincia de Buenos Aires4, GeoDesia4 y Foto Interpretación -Google Earth-.

Una vez clasificadas las manzanas del partido según estos dos criterios, se logran identificar las tierras vacantes (de una manzana o más), como así también las zonas con gran cantidad de parcelas vacantes de menor tamaño, las cuales se denominarán zonas en vías de consolidación. A su vez, este análisis permite visualizar en dónde están insertas, los usos cercanos principales y los tipos residenciales predominantes de la zona (Figuras $\underline{2}$ y $\underline{3}$ ). 
Figura 2. Clasificación según intensidad de ocupación

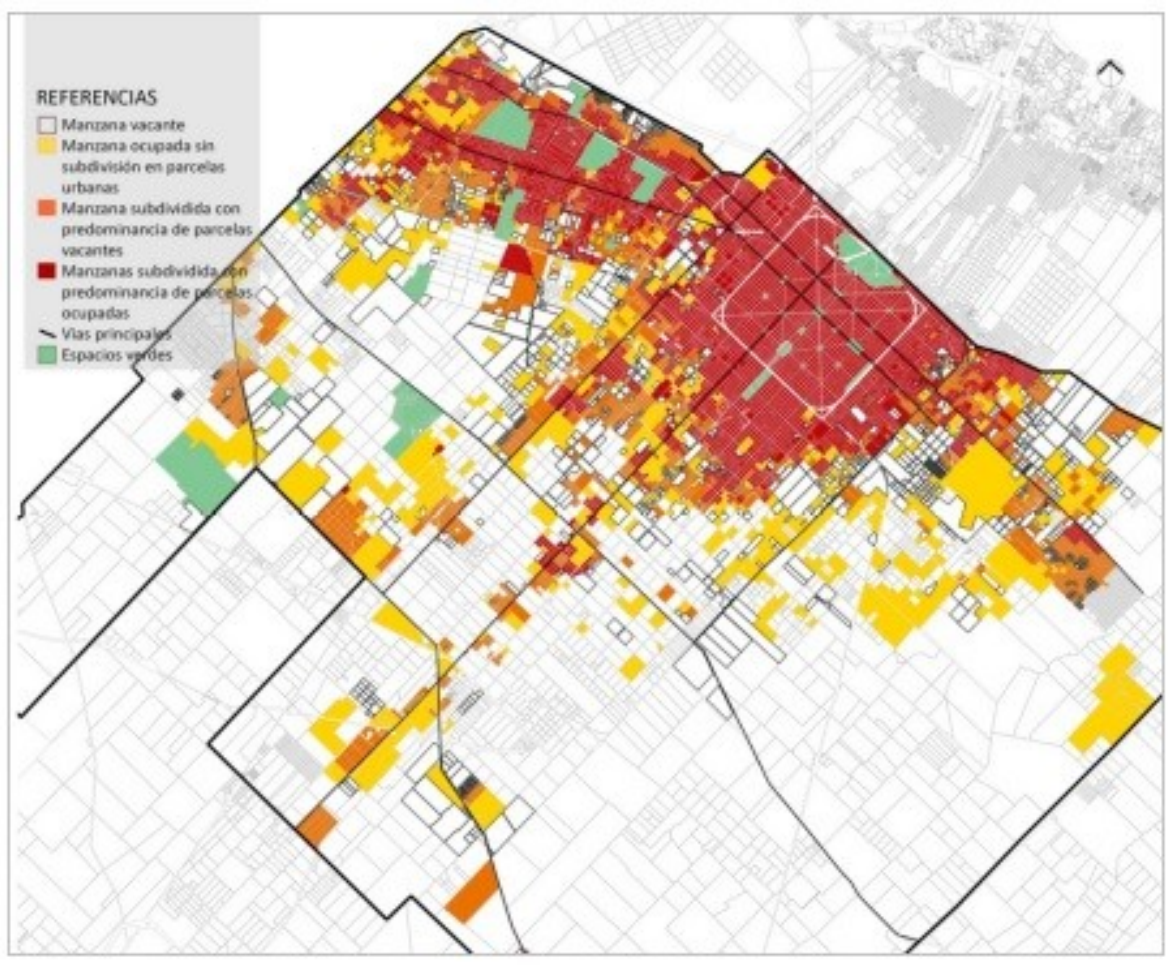

Fuente: Elaboración propia

En cuanto al uso potencial, es necesario analizar el marco legal que rige los usos de suelo en el Municipio. El Código de Ordenamiento Urbano (COU), vigente desde el año 2010 en el Partido de La Plata, clasifica el territorio en “áreas”, las cuales son ámbito resultante de la primera división que se hace sobre el territorio del Partido, con el fin de ordenar en forma general los distintos usos que en él se desarrollan (Figura 4). A efecto del presente análisis, se explican a continuación las tres áreas (Tabla 4):

- Área urbana: territorio destinado al asentamiento poblacional intensivo, con uso predominante residencial, actividades terciarias, producción compatible, equipamientos y servicios comunitarios. Está conformada por las zonas centrales, residenciales y de articulación. 
Figura 3. Clasificación según tipo predominante de uso (por manzana)

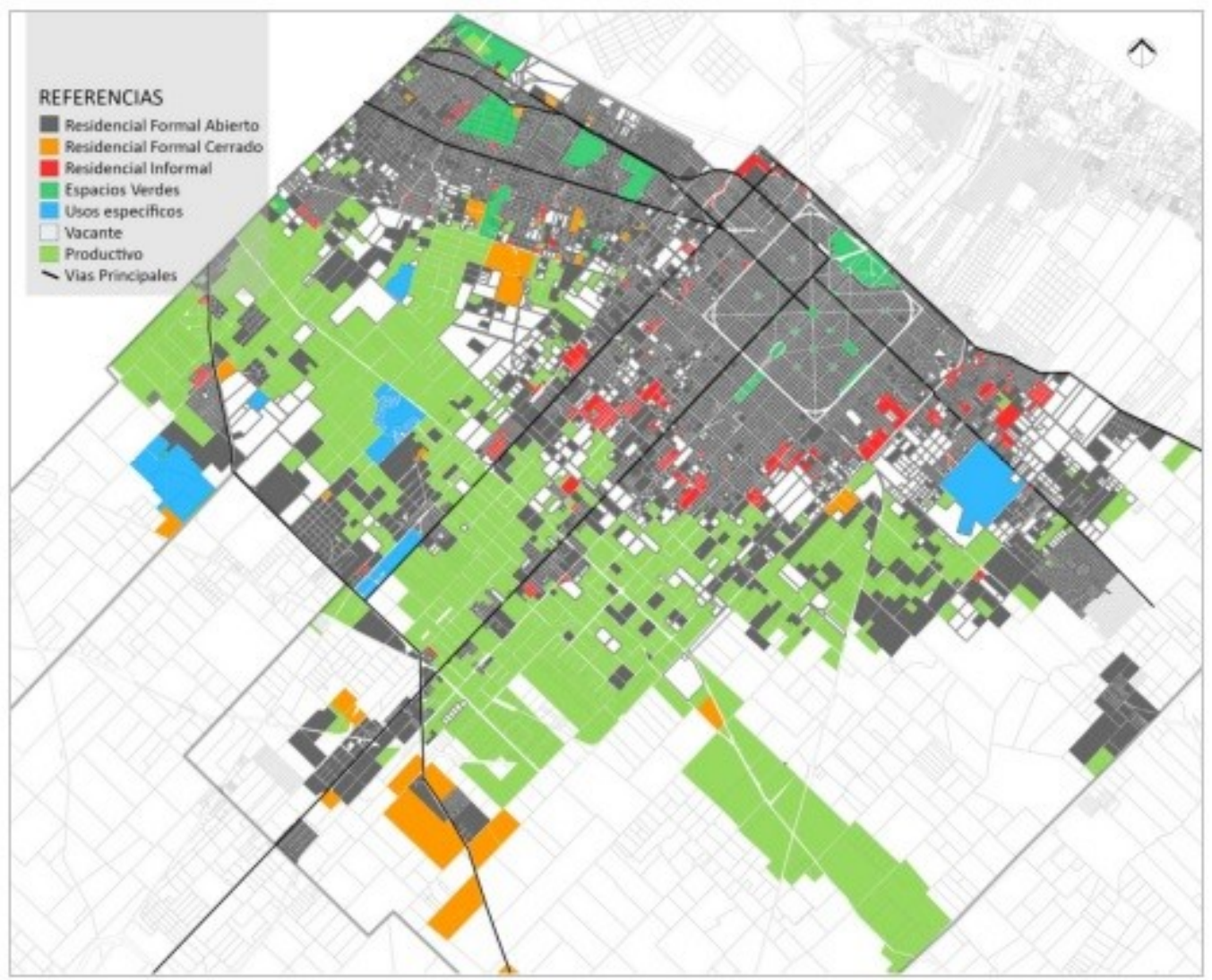

Fuente: Elaboración propia

- Área complementaria: comprende los sectores adyacentes o próximos al área urbana considerados como zonas de ampliación o ensanche urbano. Está conformada por las zonas de Reserva Urbana, Industriales Mixtas y Corredores de Servicio.

- Área rural: ámbito territorial destinado a la localización de usos agropecuarios de tipo intensivo y extensivo, con explotaciones hortícolas, ganadería de cría, tambos y agricultura extensiva y actividades forestales, extractivas e industriales. Admite la vivienda permanente aislada, el uso habitacional unifamiliar y Proyectos Especiales o Particulares.

Tabla 4. Áreas del COU según eje de crecimiento

\begin{tabular}{|c|c|c|c|c|c|c|c|c|c|c|}
\hline & \multicolumn{2}{|c|}{$\begin{array}{c}\text { Área } \\
\text { urbana }\end{array}$} & \multicolumn{2}{c|}{$\begin{array}{c}\text { Área } \\
\text { complementaria }\end{array}$} & \multicolumn{2}{c|}{ Área rural } & \multicolumn{2}{c|}{ Total } & \multicolumn{2}{c|}{$\begin{array}{c}\text { Área urbanizable } \\
\text { (urbana + compl) }\end{array}$} \\
\hline & (ha) & $\mathbf{\%}$ & $\mathbf{( h a )}$ & $\mathbf{\%}$ & $\mathbf{( h a )}$ & $\mathbf{\%}$ & $\mathbf{( h a )}$ & $\mathbf{\%}$ & $\mathbf{( h a )}$ & $\mathbf{\%}$ \\
\hline Eje norte & 6739 & 60 & 1677 & 15 & 2802 & 25 & 11219 & 100 & 8416 & 75 \\
\hline Eje sureste & 2359 & 15 & 438 & 3 & 13463 & 82 & 16261 & 100 & 2797 & 18 \\
\hline Eje suroeste & 3419 & 6 & 4095 & 7 & 51086 & 87 & 58600 & 100 & 7514 & 13 \\
\hline
\end{tabular}

Fuente: Elaboración propia 
Figura 4. Áreas según Código de Ordenamiento Urbano

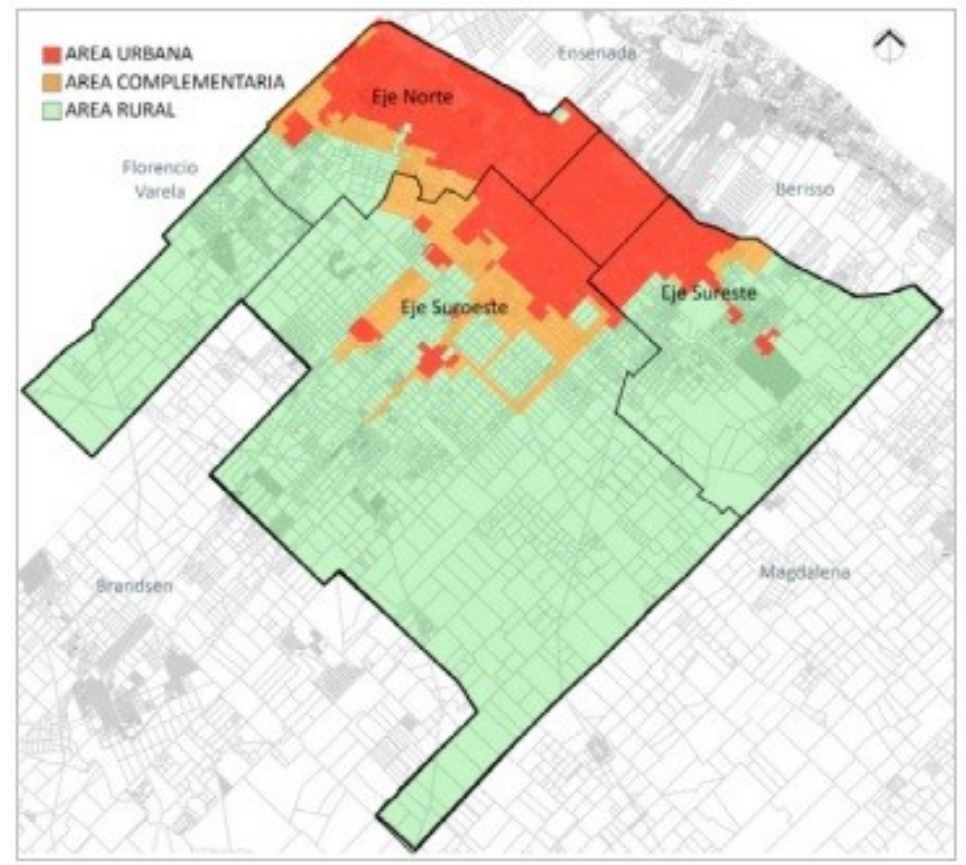

Fuente: Elaboración propia en base a urBAsig

Dentro del "área urbanizable” -área urbana y complementaria del partido-, se puede visualizar una gran cantidad de zonas en proceso de consolidación, con gran cantidad de parcelas vacantes en los tres ejes de crecimiento (Tabla 5), como así también manzanas vacantes, que van desde 517 ha en el eje sureste (24\% del área urbanizable), 1159 ha en el norte (17\% del área urbanizable), y hasta 1229 ha en el eje suroeste (19\% del área urbanizable).

Tabla 5. Formas e intensidades de ocupación dominantes, por manzana, según eje de crecimiento en área urbanizable (área urbana + área complementaria)

\begin{tabular}{|c|c|c|c|}
\hline & Eje norte & Eje sureste & Eje suroeste \\
\hline Residencial consolidado & $41 \%$ & $52 \%$ & $26 \%$ \\
\hline Residencial baja ocupación & $11 \%$ & $7 \%$ & $18 \%$ \\
\hline Productivo & $5 \%$ & $1 \%$ & $16 \%$ \\
\hline Espacio verde & $9 \%$ & $0 \%$ & $1 \%$ \\
\hline Industrial & $0 \%$ & $0 \%$ & $1 \%$ \\
\hline Residencial en consolidación & $\mathbf{1 7 \%}$ & $\mathbf{1 6} \%$ & $\mathbf{1 9} \%$ \\
\hline Vacante & $\mathbf{1 7} \%$ & $\mathbf{2 4} \%$ & $\mathbf{1 9} \%$ \\
\hline
\end{tabular}

Fuente: Elaboración propia

\section{Resultados}

Del análisis previo, donde se clasificaron las manzanas según uso e intensidad de ocupación, y 
según el área del COU en donde están insertas, se identificaron tierras vacantes en distintos contextos, lo que nos permite dar cuenta de la situación locacional de las mismas, en relación a qué usos están, sobre qué eje con determinadas características, y qué se permite hacer en ellas. A partir del análisis realizado previamente por eje de crecimiento, se han podido identificar los siguientes 6 tipos de zonas y manzanas (Tabla 6, Figura 5):

Tabla 6. Tipos de zonas y manzanas

\begin{tabular}{|c|c|c|}
\hline \multicolumn{3}{|r|}{ AREAS } \\
\hline $\begin{array}{c}\text { Tipo } \\
1\end{array}$ & $\begin{array}{l}\text { Zona con gran cantidad de } \\
\text { parcelas vacantes en } \\
\text { entorno informal }\end{array}$ & $\begin{array}{c}\text { Zonas que agrupan varias manzanas con la misma } \\
\text { condición, parceladas y presentando gran cantidad de } \\
\text { pequeños lotes vacíos, en entornos de urbanizaciones } \\
\text { informales (villas y asentamientos). }\end{array}$ \\
\hline Tipo & $\begin{array}{l}\text { Zona con gran cantidad de } \\
\text { parcelas vacantes en } \\
\text { entorno formal cerrado }\end{array}$ & $\begin{array}{c}\text { Zonas que agrupan varias manzanas con la misma } \\
\text { condición, parceladas y presentando gran cantidad de } \\
\text { pequeños lotes vacíos, en entornos de urbanizaciones } \\
\text { cerradas. }\end{array}$ \\
\hline Tipo & $\begin{array}{l}\text { Zona con gran cantidad de } \\
\text { parcelas vacantes en } \\
\text { entorno formal abierto }\end{array}$ & $\begin{array}{c}\text { Zonas que agrupan varias manzanas con la misma } \\
\text { condición, parceladas y presentando gran cantidad de } \\
\text { pequeños lotes vacíos, en entornos de residencias formales } \\
\text { abiertas. }\end{array}$ \\
\hline \multicolumn{3}{|r|}{ MANZANAS } \\
\hline $\begin{array}{c}\text { Tipo } \\
4\end{array}$ & $\begin{array}{l}\text { Manzanas vacantes en área } \\
\text { urbana }\end{array}$ & $\begin{array}{l}\text { Manzanas vacantes localizadas en áreas urbanas, } \\
\text { delimitadas por la normativa de usos de suelo. }\end{array}$ \\
\hline Tipo & $\begin{array}{l}\text { Manzanas vacantes en área } \\
\text { complementaria }\end{array}$ & $\begin{array}{l}\text { Manzanas vacantes localizadas en áreas complementarias, } \\
\text { delimitadas por la normativa de usos de suelo. }\end{array}$ \\
\hline $\begin{array}{c}\text { Tipo } \\
6\end{array}$ & $\begin{array}{l}\text { Manzanas vacantes en área } \\
\text { rural adyacente }\end{array}$ & $\begin{array}{c}\text { Manzanas vacantes localizadas en áreas rurales, adyacentes } \\
\text { a las áreas urbanizables (áreas urbanas y complementarias) } \\
\text { delimitadas por la normativa de usos de suelo. }\end{array}$ \\
\hline
\end{tabular}

Fuente: Elaboración Propia

En el denominado eje norte predominan el uso residencial formal abierto y las urbanizaciones cerradas, siendo los espacios más consolidados los que se encuentran en relación a las vías principales -exceptuando la zona ubicada al norte del eje, con grandes tierras vacantes calificadas como "área urbana” en la normativa, pero que aún no han sido ocupadas-. A su vez, muy cercanos al casco fundacional, existen grandes lotes vacantes en el medio de áreas urbanas. En la zona ubicada más al sur se localizan gran cantidad de parcelas pequeñas vacantes -pudiendo inferir que esa zona se encuentra actualmente en proceso de consolidación-, amplias zonas de "reserva urbana" que no están en relación a una vía principal de circulación, como así también parcelas rurales que mantienen usos productivos, dentro de las áreas urbanas, que se estima que en el corto o mediano plazo serán ocupadas con usos urbanos. 
Figura 5. Zonas en vías de consolidación y manzanas vacantes en el Partido de La Plata clasificadas según tipo

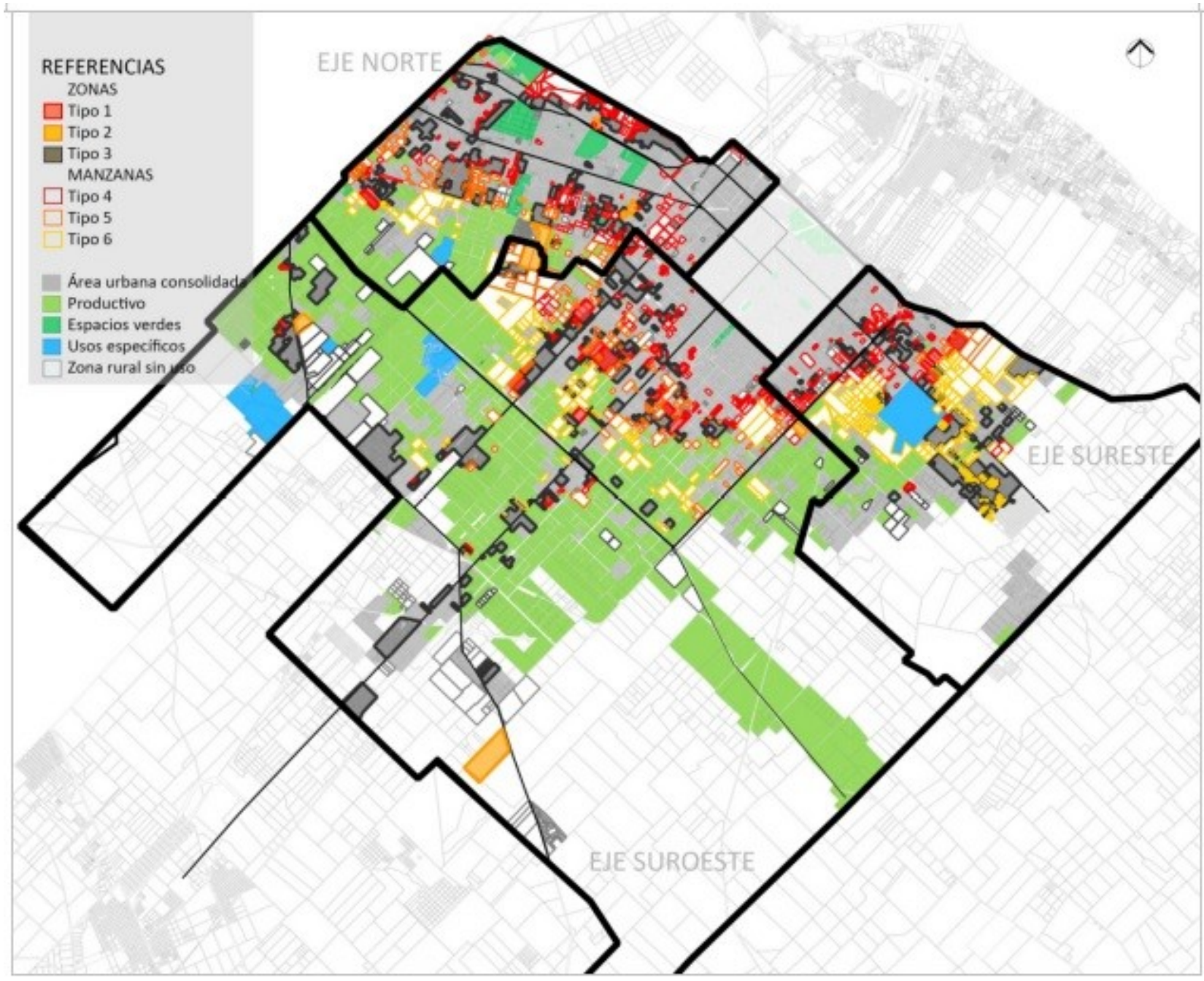

Fuente: Elaboración propia

El eje sureste, por su parte, posee características diferenciales al norte. Con gran predominio de uso residencial, y a su vez gran cantidad de asentamientos informales, posee la zona adyacente al casco fundacional muy consolidada, exceptuando dos grandes tierras vacantes al interior de la misma. A su vez, el área urbana se transforma en área rural sin transición, al cruzar la av. 90, generando que de un lado y otro de esa vía de circulación haya situaciones muy distintas, de un lado muy consolidado y del otro prácticamente todo vacante. Muchos de los grandes espacios vacantes de esta zona son canteras en desuso, que hoy se encuentran inundadas y son un riesgo para la población. Asimismo, la existencia de una urbanización al final de la av. 7 tracciona, ya que muchas de las tierras intersticiales entre ambas urbanizaciones son rurales, pero se encuentran expectantes por esta situación, aunque hoy la normativa no permita allí usos urbanos.

Por último, en el eje suroeste es donde existe la mayor cantidad de asentamientos precarios, y la expansión urbana está en relación al desborde del casco y luego a las vías principales de circulación, habiendo gran disponibilidad de tierras urbanizables en relación a estos espacios. En 
relación a las zonas en vías de consolidación, es el eje que presenta más cantidad de hectáreas de parcelas vacantes en entorno formal abierto (Tabla 7). A su vez, es el eje que presenta la mayor cantidad de tierra rural, destacándose las actividades agrícolas y frutihortícolas.

Tabla 7. Zonas en vías de consolidación, según eje de crecimiento

\begin{tabular}{|c|c|c|c|c|c|c|}
\hline & \multicolumn{2}{|c|}{ Eje norte } & \multicolumn{2}{c|}{ Eje sureste } & \multicolumn{2}{c|}{ Eje suroeste } \\
\hline Zonas Tipo 1 & 76 ha & $6 \%$ & 159 ha & $15 \%$ & 435 ha & $16 \%$ \\
\hline Zonas Tipo 2 & 74 ha & $6 \%$ & 0 ha & $0 \%$ & 318 ha & $11 \%$ \\
\hline Zonas Tipo 3 & 1052 ha & $88 \%$ & 891 ha & $85 \%$ & 2073 ha & $73 \%$ \\
\hline Total & 1202 ha & $100 \%$ & 1050 ha & $100 \%$ & 2826 ha & $100 \%$ \\
\hline
\end{tabular}

Fuente: Elaboración Propia

En relación a la distribución de las tierras según la normativa (Tabla 8), el eje norte tiene la particularidad de presentar la mayor cantidad de manzanas vacantes dentro del área urbana, a diferencia de los otros dos ejes de crecimiento. Esto significa que muchas de las tierras tienen entonces potencial de urbanizar en el corto plazo, por no necesitar grandes tiempos en trámites o cambios de usos. Por su parte, el eje sureste presenta gran cantidad de tierras vacantes en el área adyacente al área urbana; esto se debe en parte a que, como vimos en la Tabla 4, el área complementaria posee poca cantidad de hectáreas, pero a su vez la gran mayoría de ellas están vacantes.

Tabla 8. Tipos de manzanas vacantes, según eje de crecimiento

\begin{tabular}{|c|c|c|c|c|c|c|}
\hline & \multicolumn{2}{|c}{ Eje norte } & \multicolumn{2}{c|}{ Eje sureste } & \multicolumn{2}{c|}{ Eje suroeste } \\
\hline Tipo 4 & 747 ha & $51 \%$ & 287 ha & $15 \%$ & 249 ha & $11 \%$ \\
\hline Tipo 5 & 426 ha & $29 \%$ & 262 ha & $14 \%$ & 942 ha & $40 \%$ \\
\hline Tipo 6 & 301 ha & $20 \%$ & 1342 ha & $71 \%$ & 1176 ha & $50 \%$ \\
\hline Total & $\mathbf{1 4 7 4}$ ha & $\mathbf{1 0 0} \%$ & $\mathbf{1 8 9 1}$ ha & $\mathbf{1 0 0} \%$ & $\mathbf{2 3 6 7}$ ha & $\mathbf{1 0 0} \%$ \\
\hline
\end{tabular}

Fuente: Elaboración propia

\section{Conclusiones}

A partir del presente análisis, en el Partido de La Plata se evidencia un proceso de expansión en forma dispersa, pudiendo diferenciarse las características de los espacios vacantes según los ejes de crecimiento, los usos predominantes y las intensidades de ocupación, concluyendo que existe gran cantidad de tierras vacantes al interior de las periferias del partido, de grandes dimensiones (2913 ha vacantes de una manzana o más en el "área urbanizable" del partido -734 tierras-, y casi la misma cantidad en las áreas adyacentes, 2819 ha -364 tierras, sin subdividir-), y también parcelas dispersas de pequeño tamaño, en las zonas en vías de consolidación. 
Por un lado, la existencia de estas tierras contribuye a la generación de periferias fragmentadas desde varios aspectos: generan discontinuidades en la trama, disfuncionalidades para el conjunto del área urbana, como así también contribuyen a la degradación de barrios; y, al no ocuparse estos espacios contribuyen a que la ciudad se expanda cada vez más, incidiendo en el mercado de suelo y en los precios, generando una presión al Estado sobre la extensión de los servicios (agua, cloacas, electricidad, gas, transporte público y recolección de basura, entre otros), contribuyendo a las desigualdades económicas y sociales en el partido.

Pero a su vez, la disponibilidad de esta cantidad de espacios vacantes al interior del área urbanizable del partido y en sus áreas adyacentes se presenta como una oportunidad para la planificación urbana. Esas tierras podrían utilizarse de acuerdo a sus características propias y a las locacionales para intentar disminuir los procesos de fragmentación que se dan hoy en las periferias urbanas del Partido de La Plata, teniendo en cuenta que dependiendo de cómo se usen estas tierras en un futuro -y quiénes las usen-, se puede contribuir a que se profundicen los procesos, o que se reviertan, contribuyendo así a una ciudad más integrada.

Por último, y aún sin desconocer las limitaciones del Estado para incidir en el destino del suelo vacante -teniendo en cuenta el rol de la propiedad privada en la definición del destino de estas tierras-, es fundamental que el Estado sea quien impulse estrategias que favorezcan la transformación del área urbana en beneficio de todos los habitantes, estableciendo criterios para el uso racional del suelo. Es por ello que el aporte del presente trabajo reside en cuantificar y dimensionar la problemática de la vacancia del suelo en el Partido de La Plata, con el fin de tener herramientas para la intervención sobre la misma y formular estrategias en pos de reorientar el actual proceso de expansión urbana.

\section{Referencias bibliográficas}

Arteaga Arredondo, I. (2005). De periferia a ciudad consolidada. Estrategias de transformación de zonas urbanas marginales. Revista Bitácora Urbano Territorial, 1(9), 98-111. Universidad Nacional de Colombia. Bogotá, Colombia. Recuperado de: http://www.revistas.unal.edu.co/index.php/bitacora/issue/view/Enero-Diciembre\%202005/showToc Barenboim, C. (2013). Mercado inmobiliario. Normativa e impacto territorial. Rosario y su periferia. UNR Editora Editorial de la Universidad Nacional de Rosario.

Clichevsky, N. (2007). La tierra vacante revisitada. Elementos explicativos y potencialidades de utilización. Cuaderno Urbano. Espacio, cultura, sociedad. 6, 195-220. Resistencia, Argentina. Recuperado de: http://arq.unne.edu.ar/publicaciones/cuaderno urbano/cu 6/index.html

Fausto Brito, A. (2005). Desarrollo urbano equitativo en las ciudades mexicanas: Consideraciones respecto a los terrenos intersticiales vacantes. El caso del Área Metropolitana de Guadalajara (AMG). Third Urban Research Symposium of the World Bank on "Land Development, Urban Policy and Poverty Reduction”. Brasilia.

Fausto Brito, A y Rábago, J. (2001). ¿Vacíos urbanos o vacíos de poder metropolitano?. Revista 
Ciudades, 49, 33-39. RNIU, Puebla, México.

Frediani, J. (2009). Las nuevas periferias en el proceso de expansión urbana. El caso del Partido de La Plata. Revista Geograficando, 5(5), 103-125. Argentina.

Gonzalez Plazas, J.L. (2006). Caracterización socioespacial actual del Hábitat en la periferia urbana de Manizales. Revista de Arquitectura El Cable, 5, 8-25.

Larangeira, A. (2004). Tierra vacante en las ciudades de América Latina: desafíos y oportunidades. Lincoln Institute of Land Policy. Recuperado de www.lincolninst.edu

Ministerio de Infraestructura y Servicios Públicos. Social de Tierras, Urbanismo y Vivienda (2015). Registro Público Provincial de Villas y Asentamientos. Disponible en http://www.sstuv.gba.gov.ar/idehab/ [consulta julio - agosto 2016]

Municipalidad de La Plata (2010). Código de Ordenamiento Urbano del Partido de La Plata. Ordenanza 10.703.

Rocca, M.J. y Rios, L. (2012) "Procesos de expansión urbana, políticas territoriales y transformaciones emergentes. El caso de la Provincia de Buenos Aires". La Plata, Argentina. http://sedici.unlp.edu.ar/bitstream/handle/10915/27051/Proceso de expansi\%C3\%B3n urbana.pdf? sequence $=1$ [consulta julio-agosto 2016]

urBAsig. Servidor de Mapas. Ministerio de Infraestructura y Servicios Públicos http://sig.gobierno.gba.gov.ar/urbasig/ [consulta julio - agosto 2016]

UTDT-CIPUV (2013). Centro de Investigación de Políticas Urbanas y de Vivienda, Atlas de Crecimiento Urbano http://www.utdt.edu/ver contenido.php?id contenido=9267\&id item menu=18003 [consulta julio agosto 2016] 